\title{
Patients with a Normal Pressure Hydrocephalus Shunt Have Fewer Complications than Do Patients with Other Shunts
}

\author{
Schenker, Pascale ; Stieglitz, Lennart H ; Sick, Beate ; Stienen, Martin N ; Regli, Luca ; Sarnthein, \\ Johannes
}

\begin{abstract}
BACKGROUND Ventriculoperitoneal (VP) shunting is a well-established therapy for hydrocephalus. However, complications are frequent. The incidence of idiopathic normal pressure hydrocephalus (NPH) increases with the aging of the population. We evaluated the functional status of patients and the classification of complications associated with VP shunt procedures in our center. METHODS We recorded all VP shunt procedures in our prospective patient registry from January 2013 to December 2015. Functional outcome (Karnofsky Performance Status [KPS] and modified Rankin Scale) and complications were compiled from patient records. Any deviation from the normal postoperative course within 3 months after surgery was considered a complication. Complications were classified with the therapy-oriented Clavien-Dindo grading system. We evaluated potential risk factors with a logistic regression model. RESULTS From 285 procedures in the reporting period, 90 were excluded, resulting in 195 patients. Among those patients, 174 (90\%) were shunt implantations and 21 (11\%) were shunt revisions. Forty-four shunts $(23 \%)$ were implanted for NPH. Median KPS improved over the first year after surgery. Although some type of complication was observed in 114 patients (58\%), 60 of those complications $(31 \%)$ did not require surgical treatment (Clavien-Dindo grade $<3$ ). In 50 patients $(26 \%)$, the complication concerned the shunt itself. A high KPS at admission and NPH as underlying indication significantly reduced the odds ratio for a complication. CONCLUSIONS Although shunt surgery has a high general rate of complications, this rate is significantly lower for patients with NPH. The decision for shunting in patients with NPH should consider the low complication rate specific for the group of patients with NPH.
\end{abstract}

DOI: https://doi.org/10.1016/j.wneu.2017.10.151

Posted at the Zurich Open Repository and Archive, University of Zurich

ZORA URL: https://doi.org/10.5167/uzh-150982

Journal Article

Accepted Version

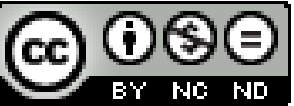

The following work is licensed under a Creative Commons: Attribution-NonCommercial-NoDerivatives 4.0 International (CC BY-NC-ND 4.0) License.

Originally published at:

Schenker, Pascale; Stieglitz, Lennart H; Sick, Beate; Stienen, Martin N; Regli, Luca; Sarnthein, Johannes (2018). Patients with a Normal Pressure Hydrocephalus Shunt Have Fewer Complications than Do 
Patients with Other Shunts. World Neurosurgery, 110:e249-e257.

DOI: https://doi.org/10.1016/j.wneu.2017.10.151 
1 Normal pressure hydrocephalus shunt patients have fewer complications than

2 other shunt patients.

3 Pascale Schenker ${ }^{1}$, Lennart Stieglitz ${ }^{1}$, Beate Sick ${ }^{2}$, Martin N. Stienen ${ }^{1}$, Luca Regli ${ }^{1}$,

4 Johannes Sarnthein ${ }^{1}$

5

61 University Hospital Zurich, Department of Neurosurgery, Zurich, Switzerland

72 University of Zurich, Epidemiology, Biostatistics and Prevention Institute (EBPI),

8 Zurich, Switzerland

9

10 Corresponding author:

11 Lennart Stieglitz

12 Department of Neurosurgery, University Hospital Zurich, University of Zurich

13 Frauenklinikstrasse 10

$14 \mathrm{CH}-8091$ Zürich, Switzerland

15 E-Mail: Lennart.Stieglitz@usz.ch

17 Running title: Complications with NPH shunts

18

19 Keywords: Clavien-Dindo classification system; complications; Normal pressure

20 hydrocephalus; outcome measures; ventriculoperitoneal-shunt surgery 

41 for a complication.

42

\section{Abstract}

Background: Ventriculoperitoneal (VP) shunting is a well-established therapy for hydrocephalus. However, complications are frequent. The incidence of idiopathic normal pressure hydrocephalus (NPH) rises with the aging of the population. We evaluated the functional status of patients and the classification of complications associated with VP shunt procedures in our center.

Methods: We recorded all VP-shunt procedures in our prospective patient registry from January 2013 to December 2015. Functional outcome (KPS and mRS) and complications were compiled from patient records. Any deviation from the normal postoperative course within 3 months after surgery was considered a complication. Complications were classified with the therapy-oriented Clavien-Dindo classification system (CDG). We evaluated potential risk factors with a logistic regression model.

Results: From 285 procedures in the reporting period, 90 were excluded, resulting in 195 cases. Among those, 174 (90\%) were shunt implantations and 21 (11\%) were shunt revisions. 44 shunts (23\%) were implanted for NPH. Median KPS improved over the first year after surgery. While any type of complication was observed in 114 cases (58\%), 60 (31\%) of those complications did not require surgical treatment (CDG <3). In 50 cases (26\%) the complication concerned the shunt itself. A high KPS at admission and NPH as underlying indication significantly reduced the odds ratio

Conclusion: While shunt surgery has a high general rate of complications, this rate is significantly lower for NPH patients. The decision for shunting in NPH patients should consider the low complication rate specific for the NPH patient group. 
Introduction

46 Cerebrospinal fluid (CSF) diversion is a well-established therapy to treat various

47 causes of hydrocephalus. Idiopathic normal pressure hydrocephalus (NPH) is

48 considered a primary form of hydrocephalus and one of the few curable causes of

49 dementia. Secondary forms of hydrocephalus comprise malresorptive

50 hydrocephalus, e.g. following subarachnoid or intraventricular hemorrhage, trauma

51 and infections, or occlusive hydrocephalus in the context of mass lesions, aqueductal

52 stenosis and tumors (1). The three most common causes in adults are NPH,

53 malresorptive hydrocephalus and occlusive hydrocephalus $(2,3)$.

54 Both primary and secondary causes of hydrocephalus are commonly treated with a

55 CSF shunt $(1,2,4)$. Cerebrospinal fluid shunts have been used for more than 50 years. For secondary forms of hydrocephalus CSF diversion can be life-saving, while

57 dramatically reducing morbidity in patients suffering from $\mathrm{NPH}$ (2). In roughly $74 \%$ of 58 NPH patients, CSF shunting results in significant improvement of clinical symptoms $59(1,5,6)$.

60 Still, complications in CSF shunt procedures are common and a significant cause of

61 morbidity. As seen in studies with sufficiently long follow-up after primary shunting,

62 more than half of patients require one or multiple shunt revision procedures (7).

63 Complications are usually due to dysfunctions of the catheter, rather than related to

64 the surgical intervention, which is a short and simple procedure (8). The most

65 common complications are mechanical dysfunctions and infections. In a previous

66 study, the rate of mechanical shunt malfunctions requiring a secondary intervention

67 was $41 \%$, and $7 \%$ of cases developed a shunt infection (2). 
68 We hereby report a study on patients with hydrocephalus who underwent CSF

69 shunting in our department. We were interested to analyze how the functional

70 outcome and the complications differed between patients suffering from a NPH and

71 patients suffering from other forms of hydrocephalus. We graded complications by a

72 therapy-oriented classification scheme (9). We evaluated factors associated with an

73 increased probability for complications of shunt-related procedures. The ultimate aim

74 was to identify patients with a particularly high or low risk of complications following

75 CSF shunting. 


\section{Methods}

\section{The patient registry}

78 Our department has established a method of recording patient data, including

79 outcome parameters and complications, following a strict protocol in a patient registry

80 (10). Demographic and clinical data are collected prospectively in electronic case

81 report forms (eCRFs) at admission, after surgery, at discharge, and at each follow-up

82 visit. In case of missing data, the forms were completed with information from the

83 electronic patient records. Clinical data include the Karnofsky Performance Status

84 Scale (KPS) and the modified Rankin Scale (mRS). Demographic data include age,

85 sex, and source of admission, i.e. the place of residence before hospitalization.

\section{Patient selection criteria}

87 For this study, we retrieved records for all cases having received a shunt from

88 January 2013 to December 2015. We excluded those shunt revisions where the

89 shunt system was implanted before our reporting period or in another hospital.

90 Cases were categorized into the three diagnosis related groups: 1) NPH, 2) other

91 forms of hydrocephalus (e.g., malresorptive or occlusive hydrocephalus), and 3)

92 shunt revision. A shunt revision was only treated as a separate case if the revision

93 surgery took place in a later hospital stay than the original shunt implantation,

94 otherwise the revision was treated as a direct complication of the first intervention.

95 Follow-up was defined as the time from the first surgery until the last consultation. 


\section{Interventions}

The standard treatment of hydrocephalus in our clinic is the implantation of a ventriculoperitoneal (VP) shunt. Most of the implanted shunts had an additional gravitational valve to reduce the incidence of overdrainage events (6). If a shunt revision was needed, the shunt was replaced either in part or in total.

Before surgery, patients were scanned for symptoms of infection using Overturf's criteria (vomiting, fever, central nervous system dysfunctions and CSF leukocytosis) (11).

During surgery, antibiotic prophylaxis (Cefazolin $1.5 \mathrm{~g}$ i.v. single dose) was given to every patient 30 minutes before skin incision.

After surgery, the patient was transferred to the intensive care unit (ICU) and to the ward after unremarkable awakening from anesthesia. Depending on their clinical condition, patients were discharged to a rehabilitation clinic, to their routine surroundings (home) or other places such as another hospital or a nursing home.

\section{Complications}

Any deviation from the normal postoperative course within three months after the intervention was defined as a complication. Sequelae and failure to cure were not counted as complications. The perioperative period included 30 days after surgery, which is common practice in our hospital. Since some complications (e.g. wound infections) arise during surgery or during the postoperative period but are only detected later, the reporting period for complications was extended to three months. 
117 Symptomatic complications concerning the shunt system were grouped into the

118 categories overdrainage, underdrainage, infection, obstruction, abdominal dislocation

119 or disconnection of the catheter.

120 All complications were classified in the therapy-oriented Clavien-Dindo classification

121 system (CDG), which considers the therapy needed to treat the complication $(9,12)$ :

$122-$ CDG-1 includes any deviation from the normal postoperative course without the 123 need of a pharmacological or surgical therapy. With this wide definition, our 124 complication rate is rather high but we avoid underreporting.

125 - For CDG-2 complications only pharmacological treatment is required.

126 - Complications necessitating surgical interventions are graded CDG-3a (without general anesthesia) or CDG-3b (under general anesthesia).

- If a complication is life-threatening and/or requires treatment in the intensive care unit (ICU), grades are CDG-4a (single-organ dysfunction) or CDG-4b (multi-organ dysfunctions), respectively.

- The patient's death within the perioperative period (i.e. until 30 days after

133 If several complications occurred in one case, for ease of handling, we only report 134 the highest CDG.

\section{Statistics}

136 A descriptive analysis was performed for all relevant variables and to illustrate the

137 unadjusted relationship between the potential predictors and the outcome

138 "complication". To model the relationship between the binary outcome and the 139 potential predictors we have used two completely different model approaches - a

140 logistic regression model and a random forest (RF) classifier (13). The logistic 
141 regression results are only valid if some model assumptions are fulfilled, which we

142 check by performing a residual analysis and by performing a global test of goodness

143 of fit (le Cessie - van Houwelingen - Copas - Hosmer weighted sum of squares

144 test). To get unbiased estimates we need approximately ten times more events than

145 estimated coefficients in the regression model. Since only 114 patients with

146 complication and 81 patients without complications were observed, we were limited

147 in the complexity of the model (with less than ten coefficients). Opposed to the

148 logistic regression model, a RF can handle situations with more predictors than

149 observations. Moreover, a RF intrinsically allows for interactions and non-linearity

150 and has only very weak model assumptions. We compared both models to judge

151 whether the logistic regression model was already sufficient to fit the data.

152 Confidence intervals $(\mathrm{Cl})$ at the $95 \%$ level were calculated where appropriate using

153 the Wilson Method. All analyses regarding the model were performed with $\mathrm{R}-\mathrm{a}$

154 language and environment for statistical computing (13).

\section{Ethics}

156 The study protocol was approved upfront by the local ethics review board (Kantonale

157 Ethikkommission KEK-ZH 2012-0244). The authors have no relevant conflicts of 158 interest. 


\section{Results}

160

161

162

163

164

165

166

167

168

169

170

171

172

173

174 (median 55y).

\section{Patient status}

\section{Clinical status scales}

177 The level of functional capacity improved over the reporting period both for patients

178 with a complication (brown) and for those without a complication (green) until three to 179 six months after shunt surgery (Figure 3). 
180 Median KPS improved from $50 \%$ at admission to $70 \%$ at three months and to $80 \%$

181 after six months. The median mRS improved from 4 at admission to 3 at discharge

182 and to 2 at three and six months.

183 At each assessment, the median KPS was higher in cases in which no complication

184 occurred compared to cases in which a complication occurred within three months.

185 Patients experiencing a complication had a worse median KPS and mRS score 186 already at admission. Similarly, the rate of previous surgery was $67 \%$ for those 187 experiencing a complication, while the rate was only $45 \%$ in patients without a 188 complication.

\section{Place of residence}

190 The most common place of residence before surgery was routine surroundings

191 (home) for both patients without $(52 / 81,64 \%)$ and those with a complication $(65 / 114$, $19257 \%$ ). The proportion of patients transferred to our institution from another hospital 193 was higher in those experiencing a complication (29/114, $25 \%)$, compared to those 194 with an uneventful course $(6 / 81,7 \%$, chi2-test $p$-value $=0.0023)$. Patients without 195 any complication were discharged to their routine surroundings (home) in most cases $196(42 / 81,52 \%)$, whereas those experiencing a complication were more frequently 197 discharged to in-patient rehabilitation $(70 / 114,61 \%)$.

\section{Indication}

199 Across all patients, complications occurred in 92 cases (47\%) until discharge and in 200114 cases (58\%) at the three months follow up. When distinguishing between the 201 indications for surgery, the complication rate was lower for NPH patients (16/44, $20236 \%)$ as compared to the other indications $(90 / 130,69 \%$, chi2-test $p$-value $=0.0002)$ 203 (Figure 4). 
204 The most common types of complications are listed in Figure 5, again distinguishing

205 between NPH patients and other indications. Complications concerning the shunt 206 itself were most frequent (50 cases, 26\%) and of these $30(15 \%)$ required a shunt

207 revision. Other frequent complications were pneumonia in 36 cases (18\%), urinary 208 tract infection in $25(13 \%)$ and epileptic seizure in 20 cases (10\%), which were rare

209 for NPH patients (Figure 5). In 19 (10\%) cases an infection such as meningitis,

210 ventriculitis or another infection of the CSF occurred. Some patients had multiple

211 complications.

\section{Classification of complications}

213 The majority of complications required pharmaceutical treatment only (CDG-2;

$21446 / 195$ cases, 24\%, Table 1). Five patients died within 30 days after shunting (CDG-

$2155 ; 3 \%)$. The overall mortality rate was 7\% (14/195) during our 3-month reporting 216 period.

217 The distribution of the CDG grades of NPH and other hydrocephalus patients are 218 presented in Figure 6.

219 Figure 7A shows the CDG distribution of the 92 cases with complications registered 220 at discharge (total complication rate: 47\%, 95\%-Cl [40\% 54\%]). The majority of 221 complications $(56 / 92=61 \%, 95 \%-\mathrm{Cl}[50 \% 71 \%])$ were treated without invasive 222 treatment (CDG-1 and CDG-2).

223 The median postoperative length of hospitalization was significantly higher in cases

224 with a complication at discharge (9 vs. 4 days, p-value $<0.001$, Mann-Whitney U-

225 test). The length of stay and the CDG grade were correlated with Spearman's 226 rho=0.46 (p-value $=0.001)$ and the linear fit had a slope of 2.5 days per increment of 227 CDG (Figure 7B). 
228 The KPS at discharge decreased with the CDG grade of the complication (Figure

$2297 \mathrm{C}$ ). The KPS and the CDG grade were correlated with Spearman's rho=-0.48 ( $p$ -

230 value $<0.001$ ) and the linear fit had a slope of $-7.9 \mathrm{KPS}$ points per increment of

231 CDG. The median KPS at discharge was higher without than with a complication (75

232 vs. 40, p-value $<0.001$, Mann-Whitney U-test).

\section{Shunt-related complications}

234 The most common shunt-related complications were overdrainage and infection,

235 followed by abdominal dislocation, obstruction, underdrainage and disconnection

236 (Table 2). Overdrainage was defined as reduction of the ICP to a level below the

237 intended, leading to typical symptoms like headache and nausea or even to

238 development of hygroma or chronic subdural hematoma. Underdrainage on the other

239 hand was defined as insufficient drainage in absence of shunt-malfunction, leading to

240 enlargement of ventricles, symptoms of hydrocephalus or insufficient resolving of the

241 underlying disorder's symptoms. Infection comprises ventriculitis, meningitis, wound

242 infections and colonization of the implanted material. Obstruction was defined as a

243 condition leading to a high flow-resistance of the shunt system due to reduction of its

244 diameter or mechanical problems. In abdominal dislocation, the catheter is either not

245 implanted in the intraperitoneal cavity correctly or dislocates from there to a

246 compartment in the subcutaneous tissue for various, mostly unknown, reasons.

247 Disconnections were disturbances of the shunt's continuity, either by loosening of the

248 catheter from the shunt valve or by rupture of the catheter due to weaknesses of the

249 material.

250 Of a total of 50 shunt problems recorded at admission, discharge or follow-up,

251 overdrainage was seen in $20(10 \%)$ cases, of which in five cases a hygroma, in three

252 cases a chronic subdural hematoma and in three cases a sinking skin flap syndrome 
253 occurred. In only four (2\%) of all cases with overdrainage the underlying etiology was

$254 \mathrm{NPH}$. Most cases with overdrainage (11/20, 55\%) only needed an adjustment of the

255 valve as therapy and were therefore graded CDG-1. Underdrainage was most often 256 graded as CDG-1 as well, unlike all other shunt-related complications. Abdominal

257 dislocation, infection and obstruction usually required revision surgery (CDG-3b).

258 Shunt infection was seen in 9 cases, two of which occurred secondary after

259 extracranial procedures or infections, and four infections were subsequent to wound

260 dehiscence. When comparing between groups, only one NPH patient had an

261 infection $(1 / 44,2 \%)$ compared to $9(9 / 130,7 \%)$ patients having received shunts for

262 other indications (Fisher exact test $p$-value $=0.45$ ).

\section{Logistic regression model}

264 With our five explanatory variables, from which three are factor variables with two or

265 three levels, we need to estimate eight coefficients if no interactions are included in 266 the model. Since we only have 81 patients without a complication we cannot afford to

267 fit a logistic regression including interaction terms. The fitted logistic regression

268 model does not violate the model assumptions and is summarized in table 3 by the 269 estimated odds ratios (OR) and the range of the corresponding 95\% confidence

270 intervals along with the p-value. As predictors we selected five main parameters,

271 where clinical experience led us to assume a possible effect on the risk of

272 complications. The adjusted associations between the predictors and the outcome

273 are in concordance with the unadjusted associations that we have observed in the

274 descriptive analysis. All following statements about the association of the different

275 predictors and the risk for complication are valid for the situation where we adjust for

276 all other predictors included in the model. 
278 Age: There is no evidence for an association with the risk for a complication.

279 Indication for surgery: The risk for a complication in a NPH patient is only $31 \%$ as

280 high as compared to a patient receiving a shunt for a different indication. We are $95 \%$

281 confident that this value lies between $13-76 \%(p=0.011)$. A chi-square-test

282 confirmed that a model including the variable "indication for surgery" fits the data

283 significantly better than a model without this variable, $p$-value $=0.03$.

284 Sex: There is no evidence for an association with the risk for a complication.

285 Source of admission: There is evidence for an association with the risk for

286 complication - especially "other care", which mostly includes nursing home and

287 rehabilitation clinic, is associated with lower risk (only $35 \%$ as high). A Chi-square-

288 test confirmed that a model including the variable "source of admission" fits the data

289 significantly better than a model without this variable, $p$-value $=0.02$.

290 KPS: For every one-step increase in the KPS score at admission, the risk to

291 experience a complication is about $3 \%$ lower (OR $0.97,95 \% \mathrm{Cl} 0.96-0.99, \mathrm{p}=0.001$;

292 also confirmed via chi-square-test, $p$-value $=0.0006)$.

293

294 Since the fitted logistic regression model does not allow for interactions and non-

295 linear associations, we have also fitted a more flexible RF model to our data which

296 revealed partial dependencies between the predictors and the complication risk that

297 are in concordance with the results from the logistic regression (data not shown).

298 Confirming the results with the RF model indicates that results of the logistic

299 regression model are valid. 


\section{Discussion}

302 In 1935, Wilder Penfield stated "all surgeons who continue to face the hydrocephalus 303 problem require the support of fortified optimism“(14). Fortunately, today we have 304 advanced quite a bit from there, but nonetheless, hydrocephalus treatment remains 305 challenging in a relevant share of the cases and CSF shunts are still known to be 306 prone to complications. While technical problems with shunts may principally affect

307 all patients, in some indications for CSF shunts complications are more likely.

308 Patients with normal pressure hydrocephalus (NPH) can easily be separated from 309 other shunt-indications, as they don't suffer from an acute disorder like a recent 310 intracranial bleeding and are of a relatively homogenous age. Our patient registry 311 allows us to analyze the complication rates of CSF shunts at our institute and at the 312 same time distinguish between NPH patients and others for the sake of a better 313 understanding of the patient groups' individual risk profiles.

314 In our study, we made a direct comparison between shunting procedures for NPH

315 patients, patients with other indications for CSF shunts, and shunt revisions. In 316 general, we found the expected high rate of complications (60\%) after CSF shunt 317 procedures, where a complication was defined as any deviation from the uneventful 318 postoperative course within 3 months after surgery. While complications of the shunt 319 system itself were most frequent across all patients, the rate of other complications, 320 and in particular the rate of shunt infections, was significantly lower in NPH patients.

\section{Shunt-related complications}

322 The most common adverse events at admission, discharge and after 3 months were 323 complications concerning the shunt itself. This result corresponds with a publication 324 about hydrokinetic problems of shunting, that finds complications usually related to 
325 dysfunctions of the catheter, rather than due to the surgical intervention, which is a

326 short and simple procedure (8).

327 Shunt dysfunctions can be classified into six categories: overdrainage,

328 underdrainage, infection, abdominal dislocation, obstruction and disconnection of the

329 catheter. Overdrainage and infection are the most common complications found in

330 our series. Due to these events, the hydrocephalus was not resolved and further

331 treatment was necessary.

332 Overdrainage, as the most common shunt-related complication, occurred in $15 \%$ of

333 all cases and was equally frequent for NPH and other indications. This rate is

334 consistent with a study about the hydrokinetic parameters of shunts, which shows

335 that shunting induces unavoidable and drastic differences in intraventricular

336 hydrokinetic pressure and CSF drainage. A perfect shunt needs to comply with the

337 CSF production, which is the only constant parameter at a rate of $0.35 \mathrm{ml} / \mathrm{min}(8)$.

338 Infection of the shunt system occurred in $13 \%$ of all cases. In the literature, different

339 studies use different definitions of a shunt, which results in a wide spread of infection

340 rates $(2,7,15,16)$. We here used Overturf's criteria, characterized by typical clinical

341 symptoms and signs such as vomiting, fever, central nervous system dysfunctions

342 and CSF leukocytosis. Those may vary by the site of infection, the type of pathogen

343 and the time of outbreak after the shunt placement (11). The number of infections is

344 probably overestimated with this definition because of the possible contamination

345 with skin flora at the time of surgical removal (17). Still, our infection rate is in the

346 middle of the range reported in the literature $(1,6,13,14)$. As an interesting

347 observation, albeit not reaching statistical significance, among all cases with shunt

348 infection, there was only one NPH patient, leading to a very low infection rate of $2 \%$

349 for the NPH group. 
350 Shunt revisions occur at a rate of $24 \%$ in the literature $(1,2)$, therefore our overall

351 rate of $15 \%$ is at the lower end of this range.

\section{Classification of complications}

353 Across all complications, most frequent were grades CDG-1 and CDG-2, i.e. the

354 complication, which could be treated without surgical intervention (Figure 6). Among

355 the frequent shunt-related complications, for overdrainage either the valve was

356 adjusted (CDG-1) or the shunt needed revision (CDG-3b). When comparing NPH

357 and other hydrocephalus indications, the rate of complications for each CDG grade

358 was lower or equal for the NPH patient group (Figure 6).

359 With increasing CDG the number of days spent in hospital increased also (Figure

360 7B), which reflects the design of the therapy-oriented classification system. Likewise,

361 an increase of CDG correlated with a decrease of KPS at discharge (Figure 7C). This

362 is not trivial. For example in tumor surgery, an infarction of tissue in an eloquent area

363 that does not require pharmaceutical or surgical treatment will only be graded as

364 CDG-1 while a neurological deficit due to this complication might well be very severe

365 and result in a low KPS. As another frequent example in tumor surgery, a wound

366 infection requiring surgical treatment (CDG-3b) may not pull down the KPS at

367 discharge so that there is not a linear correlation between CDG and KPS (10). It is

368 therefore remarkable that our results indicate that - at least in CSF shunt surgery -

369 CDG seems to reflect the severity of a complication quite accurately.

\section{Risk factors for the occurrence of a complication}

371 A lower general state of health is a significant risk factor for the occurrence of a

372 subsequent complication (18). To measure the state of health at admission, we 
373 analyzed the clinical status scores, the source of admission and the indication for

374 surgery as potential risk factors for the occurrence of a complication. The analysis of

375 the risk factors is explained in the result section logistic regression.

$376 \mathrm{NPH}$ patients had a lower rate of complications of only $36 \%$ compared to $69 \%$ of the

377 other patients and usually had their shunt implanted as a first intervention.

378 Patients with a complication had a lower KPS before and after the complication

379 compared to patients without a complication. This lower KPS at admission could be

380 explained by the different background of patients. Of the cases with a complication,

$38165 \%$ underwent shunt surgery as a secondary intervention. These patients had a

382 lower KPS before shunt surgery due to a trauma, a tumor or a hemorrhage, which

383 made their primary intervention necessary and secondarily led to a hydrocephalus.

384 We found that an inferior KPS score at admission is a significant risk factor for the

385 occurrence of a subsequent complication.

386 The institution from where patients were admitted was associated with the

387 occurrence of a complication. Most patients that would not experience a complication

388 were admitted to the hospital from routine surroundings (home; $64 \%$ ), whereas $25 \%$

389 of the patients with a subsequent complication were admitted from another hospital.

390 Therefore, patients admitted to our clinic from another hospital or another department

391 in our hospital carried a significantly higher risk of suffering from a complication, than

392 patients admitted from routine surroundings or other care institutions. It is

393 conceivable that the complexity of the case was higher if patients required in-patient

394 care before transfer to neurosurgery, which eventually translated into a higher

395 complication rate. 
396 Age was not a predictive risk factor for the occurrence of a complication, in line with

397 the NPH patient group having a higher median age.

\section{Limitations of our study}

399 In our study design, most parts of the data were collected prospectively, whereas

400 some data had to be compiled retrospectively from electronic patient records. While

401 the electronic patient records were complete, the data had to be transformed to the

402 scales used in the patient registry.

403 Classifying the severity of a complication by CDG is therapy-oriented and does not

404 consider the patient's subjective well-being, satisfaction with received treatment or

405 quality of life. A complication may be classified as low-grade, even though it may

406 have a severe impact on the patient. Nevertheless, we observed a strong correlation

407 between CDG grade and functional status (KPS) for our patient group.

408 Pooling all non-NPH patients into one group entails summarizing the outcome and

409 complications of patients with $\mathrm{SAH}, \mathrm{TBI}$, tumors and other pathologies. On the other

410 hand, all these categories are made up of patients who suffer from very severe

411 diseases, which stands in stark contrast to NPH patients, which mostly only suffer

412 from few comorbidities. For this reason, we find it justified to consider the risk of

413 shunt treatment in NPH completely separated from all other indications. The number

414 of patients in this study allows only limited degree of dividing patients into subgroups.

415 To reach relevant conclusions, we decided to use this rather simple differentiation

416 into NPH patients and other indications. 


\section{Conclusions}

418 Our study provides a direct comparison of patients with shunts due to NPH and

419 patients with shunts for other indications regarding functional outcome and

420 complication rates and classification of complications. While shunt surgery in general

421 has a high rate of complications in patients with hydrocephalus following

422 hemorrhage, TBI or tumors, this rate was significantly lower for NPH patients, which

423 in general suffer from less comorbidity.

424 As a secondary finding, CDG seems to be an appropriate tool not only for classifying

425 complications, but also for evaluating the complications' severity. 
Schenker

\section{Funding}

428 This research did not receive any specific grant from funding agencies in the public, 429 commercial, or not-for-profit sectors. 


\section{References}

431 1. Shprecher D, Schwalb J, Kurlan R. Normal pressure hydrocephalus: diagnosis and treatment.

1. Shprecher D, Schwalb J, Kurlan R. Normal press
Curr Neurol Neurosci Rep. 2008 Sep;8(5):371-6.

2. Korinek AM, Fulla-Oller L, Boch AL, Golmard JL, Hadiji B, Puybasset L. Morbidity of ventricular cerebrospinal fluid shunt surgery in adults: an 8-year study. Neurosurgery. 2011 Apr;68(4):98594; discussion 94-5.

3. Joswig H, Jucker D, Lavalley A, Sprenger L, Gautschi OP, Hildebrandt G, Schaller K, Stienen MN. Shunts: Is Surgical Education Safe? World Neurosurg. 2017 Jun;102:117-22.

4. Hakim S, Adams RD. The special clinical problem of symptomatic hydrocephalus with normal cerebrospinal fluid pressure. Observations on cerebrospinal fluid hydrodynamics. J Neurol Sci. 19651965 Jul-Aug;2(4):307-27.

5. Hebb AO, Cusimano MD. Idiopathic normal pressure hydrocephalus: a systematic review of diagnosis and outcome. Neurosurgery. 2001 Nov;49(5):1166-84; discussion 84-6.

6. Lemcke J, Meier U, Müller C, Fritsch MJ, Kehler U, Langer N, Kiefer M, Eymann R, Schuhmann MU, Speil A, Weber F, Remenez V, Rohde V, Ludwig HC, Stengel D. Safety and efficacy of gravitational shunt valves in patients with idiopathic normal pressure hydrocephalus: a pragmatic, randomised, open label, multicentre trial (SVASONA). J Neurol Neurosurg Psychiatry. 2013 Aug;84(8):850-7.

7. Tuli S, Drake J, Lawless J, Wigg M, Lamberti-Pasculli M. Risk factors for repeated cerebrospinal shunt failures in pediatric patients with hydrocephalus. J Neurosurg. 2000 Jan;92(1):31-8.

8. Sotelo J. The hydrokinetic parameters of shunts for hydrocephalus might be inadequate. Surg Neurol Int. 2012;3:40.

9. Dindo D, Demartines N, Clavien PA. Classification of surgical complications: a new proposal with evaluation in a cohort of 6336 patients and results of a survey. Ann Surg. 2004 Aug;240(2):205-13.

10. Sarnthein J, Stieglitz L, Clavien PA, Regli L. A Patient Registry to Improve Patient Safety: Recording General Neurosurgery Complications. PLoS One. 2016;11(9):e0163154.

11. Overturf GD. Defining bacterial meningitis and other infections of the central nervous system. Pediatr Crit Care Med. 2005 May;6(3 Suppl):S14-8.

12. Clavien PA. Targeting quality in surgery. Ann Surg. 2013 Nov;258(5):659-68.

13. R Core Team. R: A language and environment for statistical computing. Vienna, Austria: Foundation for Statistical Computing; 2016.

14. Ingraham FD, Matson DD. Studies in the treatment of experimental hydrocephalus. J Neuropathol Exp Neurol. 1948 Apr;7(2):123-43.

15. Patwardhan RV, Nanda A. Implanted ventricular shunts in the United States: the billion-dollara-year cost of hydrocephalus treatment. Neurosurgery. 2005;56(1):139-44; discussion 44-5.

16. Central Nervous System Device Infections. Infectious Disease Clinics of North America. 2012;26(1):89 - 110.

17. Bayston R, Leung TS, Wilkins BM, Hodges B. Bacteriological examination of removed cerebrospinal fluid shunts. J Clin Pathol. 1983 Sep;36(9):987-90.

18. Joswig H, Gautschi OP, El Rahal A, Sveikata L, Bartoli A, Hildebrandt G, Schaller K, Stienen MN. Cranioplasty: Is Surgical Education Safe? World Neurosurg. 2016 Jul;91:81-8. 
473 Figure 1. Study profile and patient selection.

474 A total of 285 shunt-related procedures were performed in our unit. In the reporting 475 period, complete data were available for 195 cases. Among them, $174(90 \%)$ were 476 ventriculoperitoneal (VP) shunt implantations and 23 (11\%) were shunt revisions.

477 Figure 2. Patient age distribution.

478 The age of normal pressure hydrocephalus (NPH) patients was close to their median 479 (75 years). Other hydrocephalus patients were younger with a median age of 55 years. Shunt revision occurred more frequently with age. Lines were shifted for better 481 visibility.

Figure 3. Functional status is reduced in patients with complications.

483 Functional performance (KPS) improved within one year after shunting for patients 484 with complications (brown boxes) and without complications (green boxes). The 485 width of the bars reflects the number of patients in each group. Patients who 486 experience a postoperative complication have a lower KPS already at admission. At 48724 months follow-up, only patients with complications were seen, which is reflected in 488 the lower median KPS. The analysis of complications was done in the reporting period from discharge to 3 months (3M).

490 Figure 4. The incidence of complications is lower for NPH.

491 Complication rates are compared between the three indications for surgery, which 492 are normal pressure hydrocephalus (NPH), other hydrocephalus and revision. The 493 column height is normalized to the number of patients within each group and the 494 column width indicates the relative number of patients in each group. The 495 complication rate is lowest for NPH patients (16/44=36\%).

Figure 5. Relative frequency of classes of complications compared for NPH and other hydrocephalus.

498 Shunt-related complications occurred most often, followed by pneumonia, urinary

499 tract infection and central nervous system (CNS) infection. Normal pressure

500 hydrocephalus (NPH) patients had fewer complications than patients with an

501 occlusive or malresorptive hydrocephalus. Some patients had multiple complications. 
502 Figure 6. The severity of complications is lower for NPH.

503 The rate of complications with Clavien-Dindo Grade (CDG) $>1$ were lower for patients

504 with normal pressure hydrocephalus (NPH).

505 Figure 7. CDG grade correlates with KPS and length of stay.

506 (A) Distribution of Clavien-Dindo Grades (CDG) of complications at discharge. The

507 majority of complications $(56 / 92=61 \%, 95 \%-\mathrm{Cl}[50 \% 71 \%])$ were treated without

508 invasive treatment (CDG-1 and CDG-2).

509 (B) The length of stay is prolonged with the CDG of the complication at discharge.

510 The length of stay and the CDG were correlated with Spearman's rho $=0.46$ ( $p$-value

$511=0.001)$ and the linear fit had a slope of 2.5 days per increment of CDG. The median

512 postoperative length of hospitalization was significantly higher in cases with a

513 complication at discharge (9 vs. 4 days, $p$-value $<0.001$, Mann-Whitney U-test).

514 (C) The KPS at discharge decreases with the CDG of the complication. The KPS and

515 the CDG were correlated with Spearman's rho $=-0.48(p$-value $<0.001)$ and the linear

516 fit had a slope of -7.9 KPS points per increment of CDG. The median KPS at

517 discharge was higher without a complication (no AE, KPS $=75$ ) than with a

518 complication (CDG 1-5, KPS $=40$, p-value $<0.001$, Mann-Whitney U-test). 
520 Table 1. Clavien-Dindo Grade (CDG) of complications and the time of 521 occurrence.

522 In patients where more than one complication occurred, only the worst CDG is listed 523 for ease of handling. $\mathrm{Cl}=$ confidence interval.

\begin{tabular}{llllll}
\hline CDG & Discharge & 6 weeks & 3 months & $\begin{array}{l}\text { Surgery } \\
\text { until } \\
\text { months }\end{array}$ & $35 \%-\mathrm{Cl}$ \\
1 & 12 & 3 & 1 & $14(7 \%)$ & $4 \%-12 \%$ \\
2 & 44 & 7 & 4 & $46(24 \%)$ & $18 \%-30 \%$ \\
$3 \mathrm{a}$ & 4 & 0 & 1 & $5(3 \%)$ & $1 \%-6 \%$ \\
$3 \mathrm{~b}$ & 23 & 9 & 9 & $39(20 \%)$ & $15 \%-26 \%$ \\
$4 \mathrm{a}$ & 5 & 0 & 0 & $4(2 \%)$ & $0 \%-3 \%$ \\
$4 \mathrm{~b}$ & 0 & 0 & 1 & $1(1 \%)$ & $0 \%-3 \%$ \\
5 & 4 & 1 & 0 & $5(3 \%)$ & $1 \%-6 \%$ \\
total & 92 & 20 & 16 & $114(58 \%)$ & $51 \%-65 \%$ \\
\hline
\end{tabular}

524

525 Table 2. Shunt-related complications and Clavien-Dindo Grade (CDG).

\begin{tabular}{|c|c|c|c|c|c|}
\hline \multirow[t]{2}{*}{ Complication } & \multicolumn{4}{|c|}{$C D G$} & $\mathrm{n}$ \\
\hline & 1 & 2 & $3 a$ & $3 b$ & \\
\hline overdrainage & 11 & 2 & 1 & 6 & 20 \\
\hline underdrainage & 2 & - & - & 1 & 3 \\
\hline infection & 1 & - & - & 8 & 9 \\
\hline abdominal dislocation & - & - & - & 6 & 6 \\
\hline blocking & - & - & - & 5 & 5 \\
\hline disconnection & 1 & - & - & 1 & 2 \\
\hline total & 15 & 2 & 1 & 27 & 45 \\
\hline
\end{tabular}




\section{Table 3. Logistic regression}

528 In case of continuous predictors, the odds ratio (OR) indicates the change of risk if

529 the predictor changes by one unit. In case of categorical predictors, the OR indicates

530 the change of risk if the predictor changes from the reference level (indicated after

531 "vs.") to the mentioned level (indicated in front of "vs."). The group other

532 hydrocephalus and a lower Karnofsky Performance Status (KPS) at admission are

533 associated with a higher risk for a complication. $\mathrm{Cl}=$ confidence interval.

\begin{tabular}{|c|c|c|c|c|}
\hline & Compared categories & $\begin{array}{l}\text { Odds ratio } \\
\text { (OR) for } \\
\text { complication }\end{array}$ & $95 \%-\mathrm{Cl}$ & $p$-value \\
\hline Intercept & & 5.13 & $1.035-27.378$ & 0.049 \\
\hline age & & 1 & $0.986-1.03$ & 0.501 \\
\hline indication & $\begin{array}{l}\text { NPH vs. } \\
\text { other hydrocephalus }\end{array}$ & 0.312 & $0.125-0.757$ & 0.011 \\
\hline indication & $\begin{array}{l}\text { Revision vs. } \\
\text { other hydrocephalus }\end{array}$ & 0.477 & $0.163-1.33$ & 0.163 \\
\hline sex & Female vs male & 1.472 & $0.778-2.813$ & 0.237 \\
\hline KPS at admission & & 0.974 & 0.959-0.989 & 0.001 \\
\hline
\end{tabular}









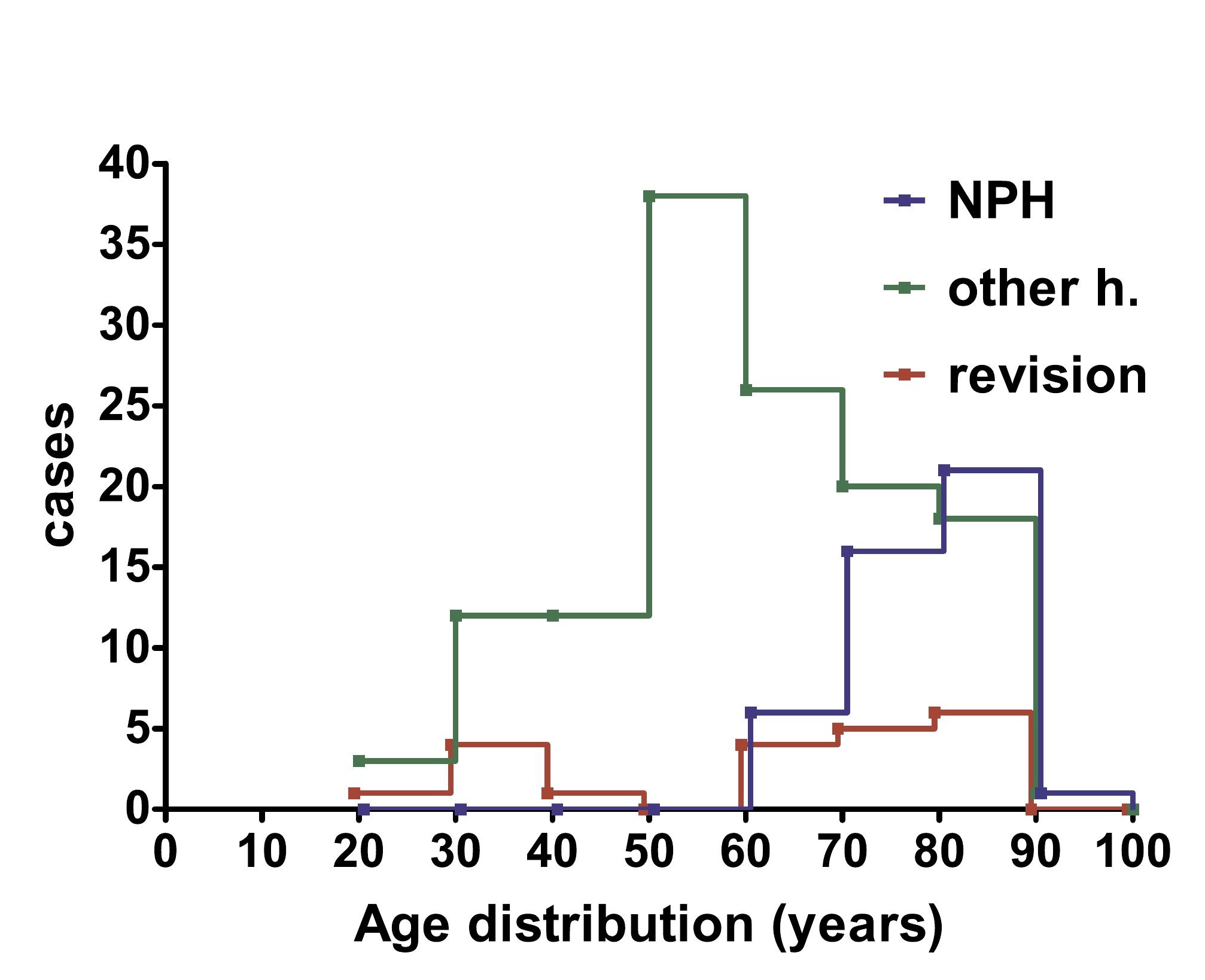

Figure 2
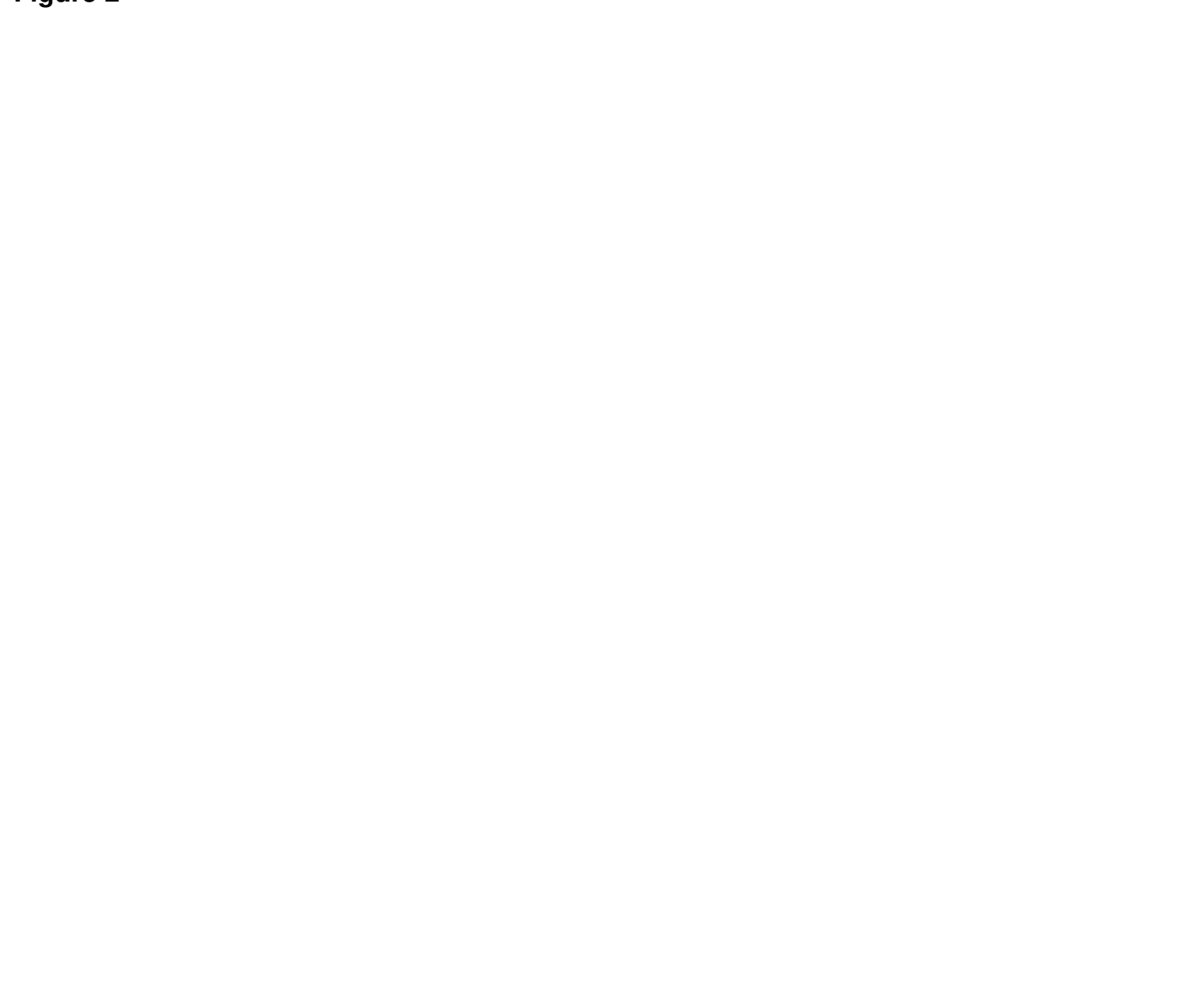
Figure 3

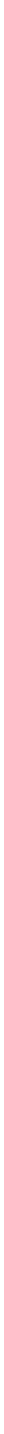

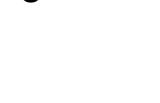

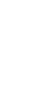

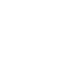
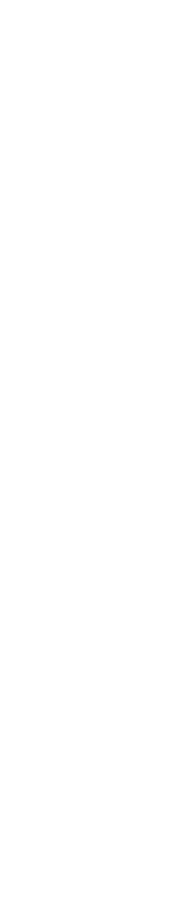

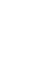
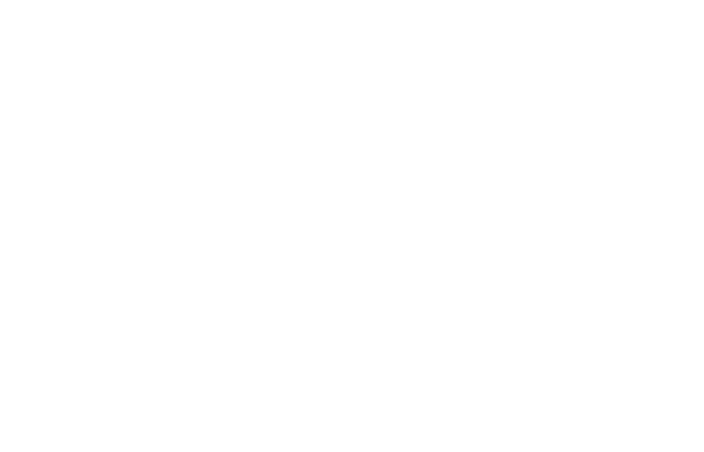

\begin{tabular}{|} 
Po \\
\hline \\
\hline
\end{tabular}


$\mathrm{NPH}$

other hydrocephalus

revision

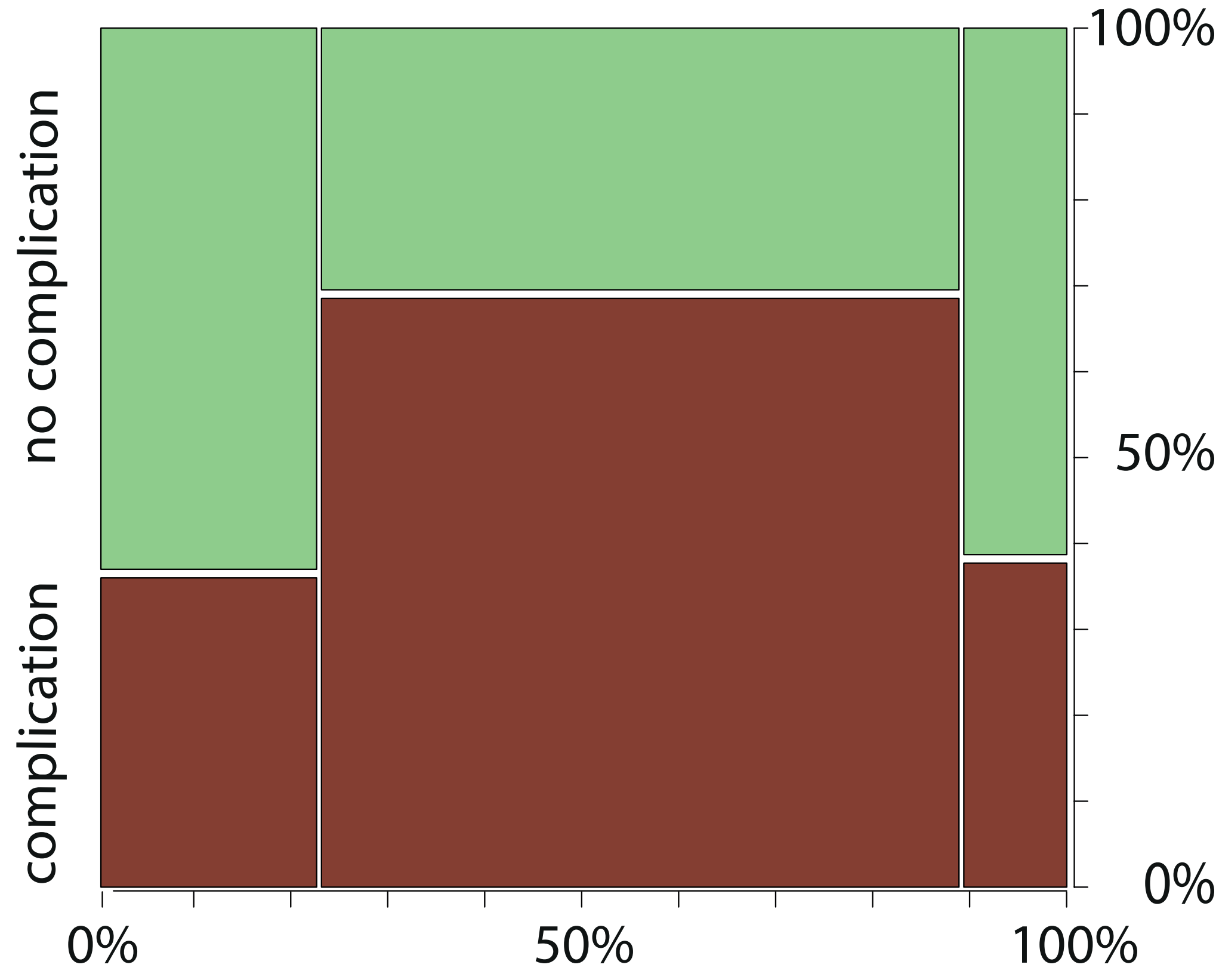

$0 \%$ $100 \%$ 
Figure 5

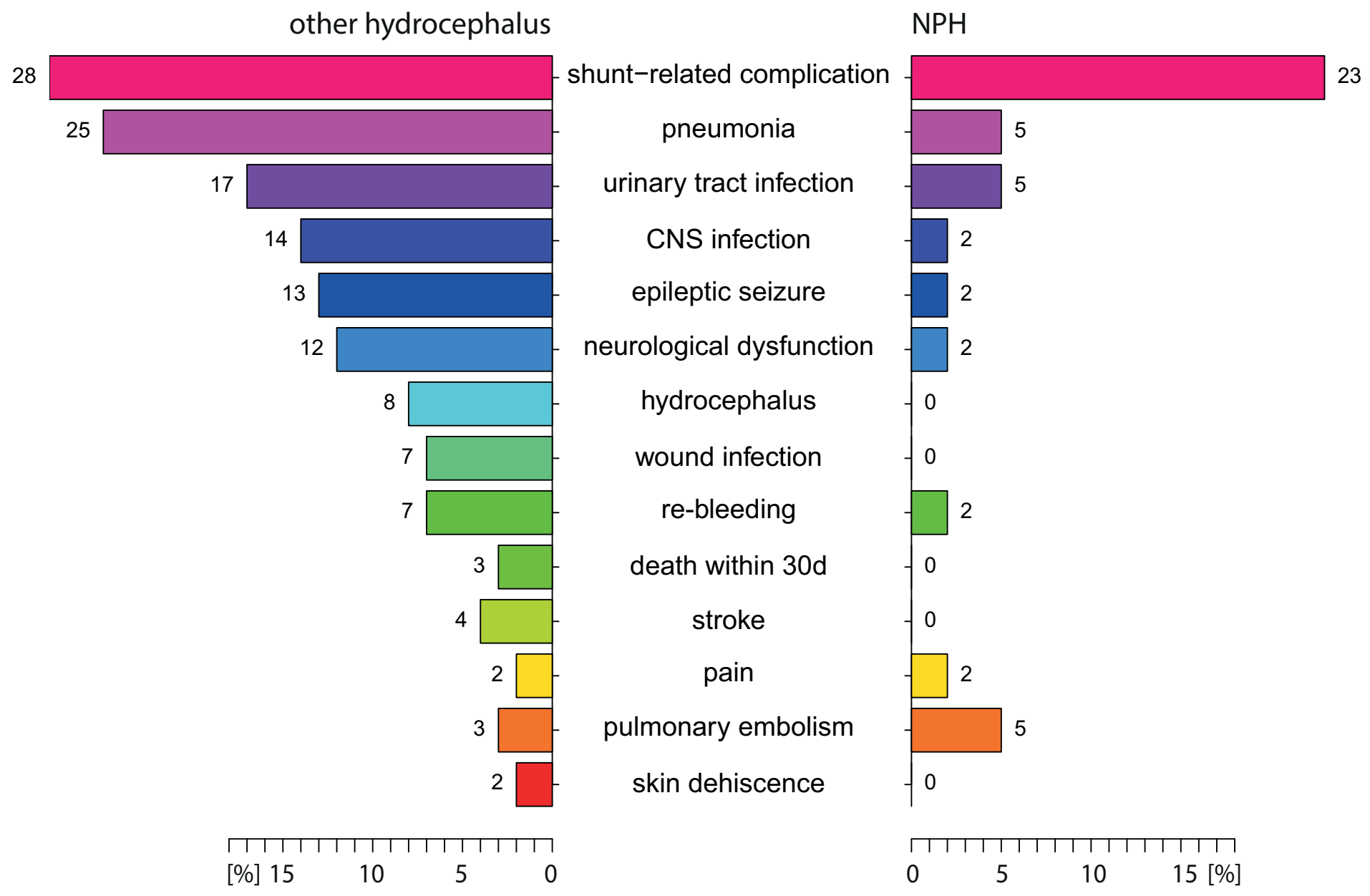

$\mathrm{NPH}$

3 


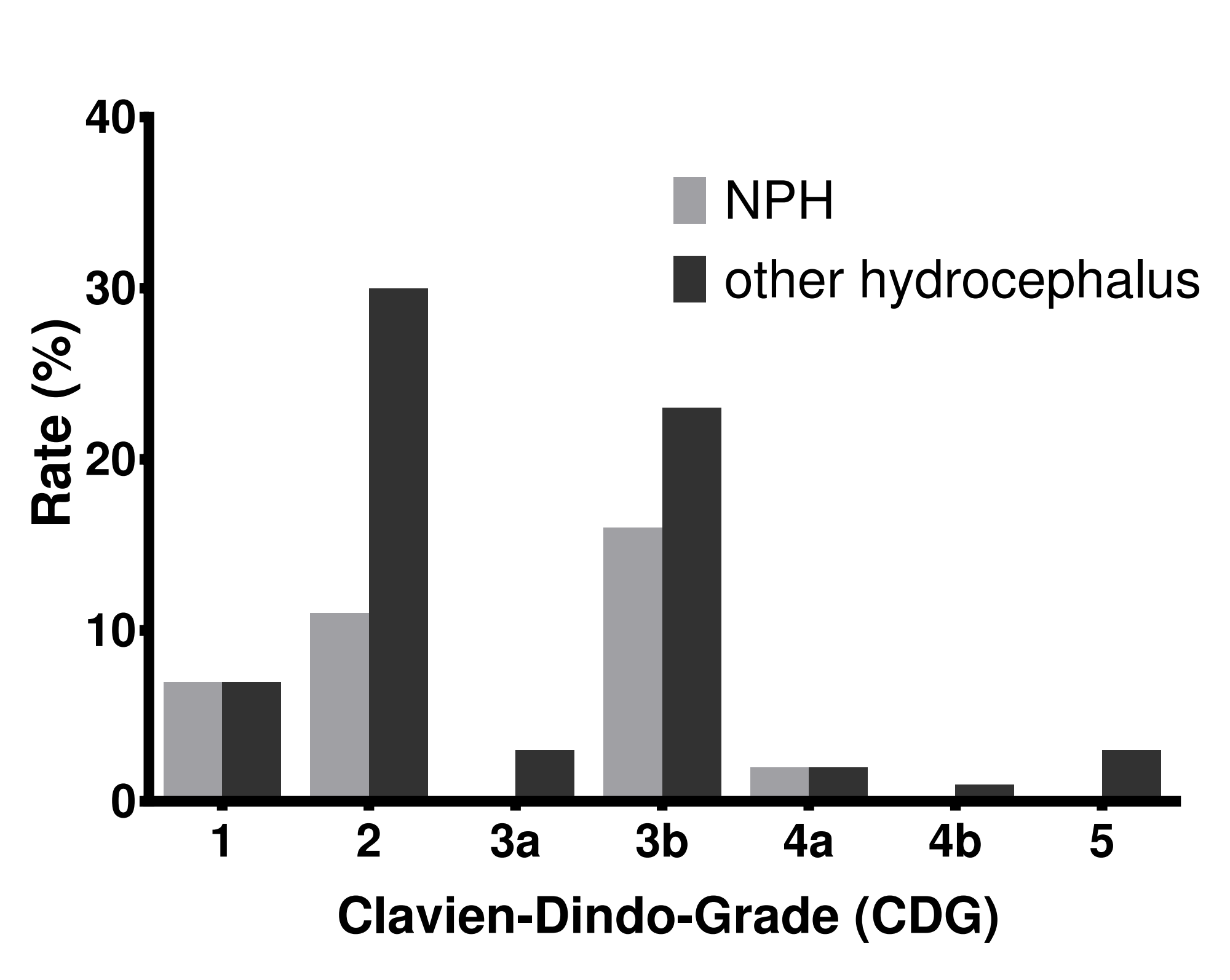

Figure 6

.

.
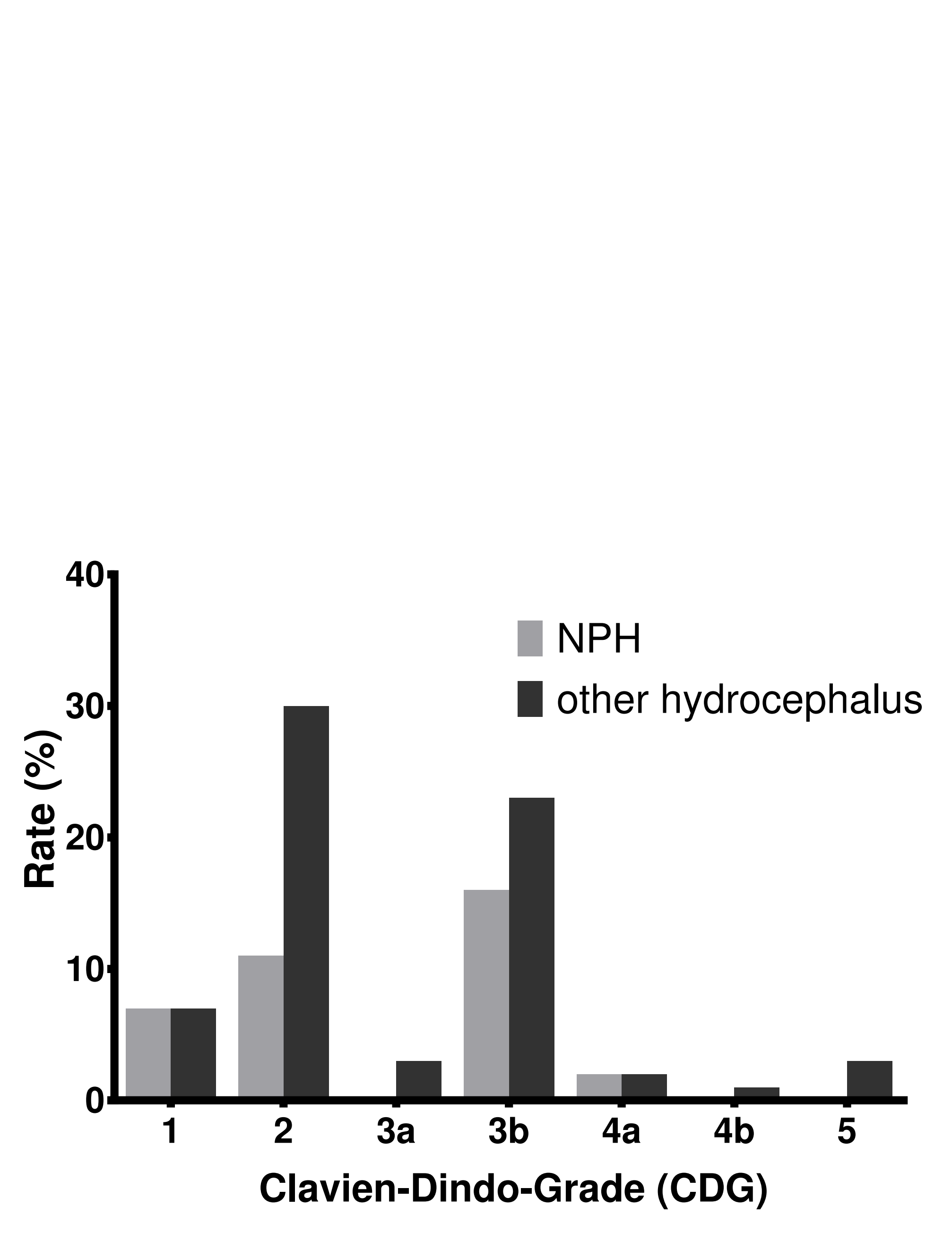
